\title{
Fütterung heranwachsender Hunde großer Rassen
}

\section{Ein Wegweiser durch den Dschungel aus Wachstumskurven, Bedarfswerten und neuen Ernährungstrends}

Cornelia Rückert, Ingrid Vervuert

Die bedarfsüberschreitende Energieversorgung während des Wachstums spielt bei der Entstehung entwicklungsbedingter Skeletterkrankungen eine große Rolle. Aufgrund des Trends, auch Junghunde mit selbst zusammengestellten Rationen zu versorgen, vergrößert sich zudem das Risiko einer Fehlversorgung mit Mineralstoffen. Der praktizierende Tierarzt wird immer häufiger mit Fragen zur optimalen Fütterung seiner Patienten konfrontiert. Im Folgenden wird auf die wesentlichen Punkte in der Fütterung von Welpen und Junghunden eingegangen, um die Beratung optimieren zu können.

\section{Problematik}

Entwicklungsbedingte Skeletterkrankungen werden vielfach bei großen Rassen vorgefunden, wobei die Genetik, aber auch Umweltfaktoren prädisponierend sein können. Aus Sicht der Fütterung spielt vor allem die bedarfsüberschreitende Energieversorgung während des Wachstums eine wesentliche Rolle. Weniger häufig sind Imbalanzen in der Mineralstoffversorgung zu beobachten. Der Trend zur Fütterung selbst zusammengestellter Rationen erhöht beim Junghund das Risiko einer Fehlversorgung mit Mineralstoffen, vor allem:

- einer Kalzium- und Kupferunterversorgung

- einer Jodüberversorgung

In der Praxis führt dies dazu, dass der Besitzer dem Tierarzt immer häufiger Fragen zur optimalen Fütterung seines Hundes stellt. Dabei geht es um die Frage, ob das derzeitige Alleinfuttermittel oder eine selbst zusammengestellte Ration nicht das Beste für den heranwachsenden Hund sei. Darüber hinaus gilt es, „Ernährungsmythen“ von zahlreichen Internetseiten aufzuklären.

\section{Wachstum und Wachstums- kontrolle}

Die Begrifflichkeit „Welpe“ wird in der Regel in Bezug zur prägenden Sozialisierungsphase verwendet. Diese beginnt mit dem Erwachen der Sinnesorganfunktionen in der 2./3. Lebenswoche und endet meist um die 16. Lebenswoche herum. Jedoch sind Welpen großer Rassen eher Spätentwickler, sodass diese auch im 5. Lebensmonat noch als Welpen bezeichnet werden können. Mit dem Beginn des Zahnwechsels schließt sich die Junghundephase an. Diese endet nicht mit der Fortpflanzungsreife, sondern vielmehr mit Erlangen der „geistigen Reife eines Adulten“.

Welpen kleiner Rassen haben ihre Hauptwachstumsphase bereits mit 6 Monaten abgeschlossen und legen sehr gleichmäßig an Körpermasse zu. Welpen großer Rassen erreichen hingegen selbst in einem Alter von 12 Monaten erst 80\% ihres Endgewichts. Zudem zeigen sie deutliche Wachstumsschübe im Zeitraum zwischen dem 3. und dem 6. Lebensmonat.

Die Wachstumsgeschwindigkeit wird maßgeblich von der Energiezufuhr be- stimmt. So zeigen Welpen und Junghunde, die ad libitum gefüttert werden, ein deutlich schnelleres Wachstum. Sie erreichen ihr Endgewicht somit schneller als ihre restriktiv gefütterten Altersgenossen. $\mathrm{Zu}$ beachten ist aber, dass die letztendlich erreichte Widerristhöhe genetisch determiniert ist und sich nicht durch die Fütterung beeinflussen lässt. Anzustreben ist also ein optimales und kein maximales Wachstum. Die Energieaufnahme sollte an die aktuelle Gewichtsentwicklung sowie an das voraussichtliche, rassetypische Endgewicht angepasst werden.

Optimale Anhaltspunkte zur Einschätzung des Ernährungszustands beim erwachsenen Hund sind leicht tastbare Rippenbögen und Dornfortsätze der Wirbelsäule sowie eine Tailleneinziehung, die seitlich und in der Aufsicht gut erkennbar ist. Um überprüfen zu können, ob ein Welpe bedarfsüberschreitend er-

\section{.konkret}

Die Gewichtsentwicklung des heranwachsenden Hundes muss dokumentiert und mit der rassetypischen

Wachstumskurve verglichen werden.

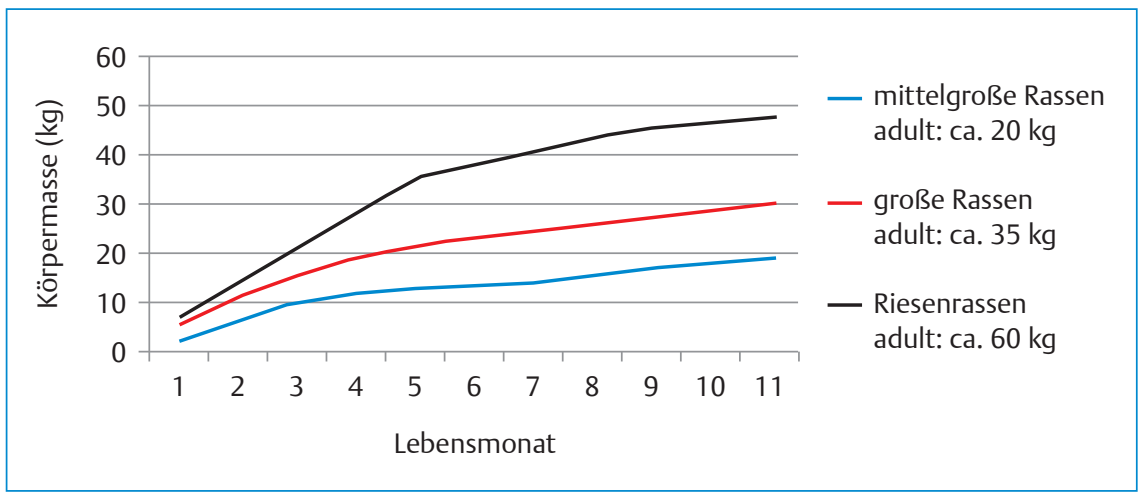

Abb. 1 Wachstumskurven verschiedener Rassen. @ C. Rückert 


\section{Gewichtsentwicklung}

Für die Entwicklung von Hunden großer Rassen gelten folgende

Richtlinien:

- mit der Vollendung des 4. Lebensmonats sollte der Welpe $1 / 3$ seines Endgewichts erreicht haben

- mit einem $1 / 2$ Jahr sollten Junghunde $60 \%$ und mit 1 Jahr $80 \%$ ihres Adultgewichts erreicht haben

nährt wird, sind diese Eckpunkte jedoch nur bedingt aussagekräftig. Die meisten Junghunde, die zu energiereich gefüttert werden, werden nicht „dick“, sondern zeigen vielmehr ein forciertes Wachstum.

Einige Beispiele für rassespezifische Wachstumskurven sind in Abb. 1 gezeigt.

\section{Energie- und Nährstoff- versorgung}

\section{Energiebedarf}

Für die Bewertung des Energiebedarfs des wachsenden Hundes ist das Wissen um dessen Haltungsform essenziell. So haben heranwachsende Hunde, die in einer Gruppe in Außenhaltung aufwachsen, einen um ca. 0,1 MJ ME/kg KM ${ }^{0,75}$ erhöhten Bedarf gegenüber Artgenossen, die einzeln im Haus gehalten werden. Der Energiebedarf ist in $>$ Tab. 1 zusammenfassend dargestellt.

\section{Proteinbedarf}

Ausgehend von ihrem deutlich längeren Wachstum haben heranwachsende Hunde großer Rassen auch erheblich länger einen erhöhten Proteinbedarf als Welpen und Junghunde kleinerer Rassen. Die Bedarfszahlen für verdauliches Rohprotein (vRp) entwickeln sich dabei wie in $>$ Tab. 2 dargestellt.

Zudem werden folgende Protein/Energie-Verhältnisse empfohlen:

- 15 g vRp/MJ ME bis zu 1/2 Jahr

- 12 g vRp/MJ ME bis zur Vollendung des 1. Lebensjahres

- 10 g vRp/MJ ME ab dem 13. Lebensmonat (für erwachsene Tiere angestrebte Relation)

Welpen und Junghunde, die einer Unterversorgung mit Protein ausgesetzt sind, fallen in der Regel nicht durch reduzierte Wachstumsraten auf. Vielmehr ist hier ein verringerter Muskelansatz zugunsten eines erhöhten Fettansatzes zu verzeichnen. Diese Tiere haben auch als

Tab. 1 Energiebedarf in MJ ME/kg KM $\mathrm{M}^{0,75} / \mathrm{d}$ in Abhängigkeit von Alter und Haltungsform [4].

\begin{tabular}{|lll|}
\hline \multirow{2}{*}{ Lebensmonat (Spanne) } & \multicolumn{2}{l|}{ Energiebedarf (MJ ME/kg KM ${ }^{0,75}$ ) pro Tag } \\
& Einzelhaltung im Haus & Gruppenhaltung, Außenhaltung \\
\hline 2.-4. & $0,7-0,8$ & $0,8-0,9$ \\
\hline 5.-6. & $0,6-0,8$ & $0,8-0,9$ \\
\hline 7.-12. & $0,5-0,6$ & $0,6-0,7$ \\
\hline
\end{tabular}

Tab. 2 Bedarf an verdaulichem Rohprotein (vRp) in $\mathrm{g} / \mathrm{kg} \mathrm{KM}^{0,75} / \mathrm{d}$ in Abhängigkeit von Alter und Körpermasse als ausgewachsener Hund [4].

\begin{tabular}{|c|c|c|c|}
\hline \multirow[t]{2}{*}{ Lebensmonat } & \multicolumn{3}{|c|}{ Proteinbedarf ( $\mathrm{g}$ vRp/kg KM $\mathrm{K}^{0,75}$ ) pro Tag } \\
\hline & $5 \mathrm{~kg} \mathrm{KM}^{*}$ & $20 \mathrm{~kg} \mathrm{KM}^{*}$ & $60 \mathrm{~kg} \mathrm{KM}$ \\
\hline 1. & 12 & 15 & 17 \\
\hline 2. & 10 & 14 & 16 \\
\hline 3. & 9 & 12 & 14 \\
\hline 4. & 8 & 10 & 12 \\
\hline 5.-6. & 7 & 8 & 10 \\
\hline 7.-12. & 5 & 6 & 6 \\
\hline
\end{tabular}

Tab. 3 Bedarf an Kalzium und Phosphor in $\mathrm{mg} / \mathrm{kg} \mathrm{KM} 0,75 / \mathrm{d}$ in Abhängigkeit vom Alter [4].

\begin{tabular}{|c|c|c|}
\hline $\begin{array}{l}\text { Lebens- } \\
\text { monat }\end{array}$ & Kalzium¹ & Phosphor $^{1}$ \\
\hline 1. & $400-582$ & $290-420$ \\
\hline 2. & $450-900$ & $290-560$ \\
\hline 3. & $470-870$ & $270-470$ \\
\hline 4. & $400-670$ & $230-270$ \\
\hline 5.-6. & $290-520$ & $170-270$ \\
\hline 7. -12. & $180-230$ & $120-150$ \\
\hline
\end{tabular}

1 Die oberen Grenzwerte gelten für Welpen großer Rassen.

Adulte eine verstärkte Neigung zu Adipositas.

Die vielfach verbreitete Meinung, eine Überversorgung mit Protein führe zu einem forcierten Wachstum, ist nur richtig, wenn die Welpen und Junghunde zeitgleich auch mit Energie überversorgt werden. In einer Studie mit Doggenwelpen konnte kein Zusammenhang zwischen einer ausschließlichen Proteinüberversorgung und Skelettfehlentwicklungen nachgewiesen werden [1].

\section{Mineralstoffe}

Die bedeutendste Rolle spielt ein ausgewogenes Ca:P-Verhältnis. Betrachtet man die hohe Wachstumsrate von Absatzwelpen, ist es nahe liegend, dass auch der Bedarf an Kalzium und Phosphor im 2. und 3. Lebensmonat am höchsten ist. Die Bedarfszahlen im Verlauf des 1. Lebensjahres sind $>$ Tab. $3 \mathrm{zu}$ entnehmen.

Beim wachsenden Hund ist ein optimales Ca: P-Verhältnis von 1,5-1,8:1 anzustreben.

Im Gegensatz dazu ist ein Ca:P-Verhältnis von 1,2:1 beim adulten Hund ausreichend. Jedoch ist nicht nur eine Unterversorgung mit Kalzium und Phosphor als kritisch anzusehen, sondern auch eine Überversorgung, z.B. infolge von Mineralstoffergänzungen zu Alleinfuttermitteln. Vielfach substituieren Besitzer zu einem Alleinfutter weitere kalziumreiche Ergänzungen (z.B. Welpenkalk), um „die Knochen zu stärken“. 


\section{Berechnung des Energiegehalts}

Der Energiegehalt darf auf den angebotenen Alleinfuttermitteln nicht deklariert werden. Daher kann es nötig sein, diesen zu berechnen und die tägliche Energieaufnahme des Hundes zu steuern. Das Vorgehen ist hierzu kurz skizziert [4]:

1. Berechnung des Bruttoenergiegehalts (GE) GE $(M \mathrm{M} / 100 \mathrm{~g})=0,02385 \times$ Rohprotein + 0,03934 $\times$ Rohfett + 0,01717 $\times \mathrm{N}$-freie Extraktstoffe $+0,01717 \times$ Rohfaser

2. Schätzung der scheinbaren Verdaulichkeit (sV) der Bruttoenergie (GE) anhand des Rohfasergehalts (Rfa) in der Trockensubstanz (TS) sV GE $(\%)=91,2-1,43 \times$ Rfa (\% TS)

3. Berechnung der verdaulichen Energie (DE) $\mathrm{DE}=\mathrm{GE} \times \mathrm{sVGE}(\%) / 100$

4. Berechnung der umsetzbaren Energie (ME) $\operatorname{ME}(\mathrm{MJ} / 100 \mathrm{~g})=\mathrm{DE}-0,00434 \mathrm{MJ} \times \mathrm{Rp}(\mathrm{g} / 100 \mathrm{~g})$

Massive Gliedmaßenfehlstellungen können die Folge sein ( $\triangleright$ Abb. 2 ).

\section{Spurenelemente}

Es sollte vor allem eine ausreichende Supplementierung von Kupfer, das eine wesentliche Rolle im Bindegewebsstoffwechsel spielt, und Zink als wichtiges Coenzym erfolgen. Bei einer exzessiven Kalziumversorgung sind auch hier Mängel zu erwarten, da die intestinale Resorptionsrate von Kupfer und Zink (2-wertige Ionen) durch den Kalziumüberschuss gestört sein kann. Der Bedarf an Kupfer liegt während des Wachstums bei $0,76 \mathrm{mg} / \mathrm{kg} \mathrm{KM}^{0,75}$, der an Zink bei $6,84 \mathrm{mg} / \mathrm{kg} \mathrm{KM} \mathrm{M}^{0,75}$.

\section{Essenzielle Fettsäuren}

Es sollte darauf geachtet werden, dass die Versorgung mit den für den Hund essenziellen Fettsäuren Linolsäure (1\% in der Trockensubstanz) und $\alpha$-Linolensäure ( $4 \%$ in der Trockensubstanz) sichergestellt ist.

\section{Rationsgestaltung}

Der Zeitpunkt, um mit der Zufütterung der Welpen zu beginnen, richtet sich nach der Wurfgröße und der Milchmengenproduktion der Mutterhündin. Meist wird mit dem Ende der 3. Lebenswoche der Welpen begonnen, erstes Beifutter wie Hackfleisch, Quark und Haferflocken anzubieten. Dieses sollte zu Beginn annähernd körperwarm sein und in breiartiger Konsistenz in flachen Schalen bereitgestellt werden. In der 7 . oder 8 . Le- benswoche werden die Welpen in der Regel vom Muttertier abgesetzt.

Eine bedarfsdeckende Versorgung eines heranwachsenden großrassigen Hundes ist sowohl über ein kommerzielles Alleinfuttermittel wie auch mit selbst zusammengestellten Rationen möglich.

\section{Alleinfuttermittel}

Die sicherlich einfachste und am wenigsten aufwendigste Variante, ihren heranwachsenden Hund bedarfsdeckend zu versorgen, stellt für Hundehalter die Fütterung eines kommerziellen Alleinfuttermittels für Welpen bzw. Junghunde dar. Dieses muss laut Definition den Bedarf des Welpen/Junghunds mit allen Nährstoffen vollständig decken.

Ob die Wahl auf ein Trocken- oder Feuchtfuttermittel fällt, bleibt dabei den persönlichen Vorlieben des Besitzers überlassen. $\mathrm{Zu}$ bedenken ist jedoch die Tatsache, dass heranwachsende Hunde einer starken Nahrungsprägung unterliegen können. Sie können im Erwachsenenalter ein Trockenfutter verweigern, wenn sie in den ersten Lebensmonaten allein mit Feuchtfutter großgezogen wurden. Zudem ist gerade die ausschließliche Fütterung von Welpen und Junghunden großer Rassen mit Feuchtfutter ein nicht unerheblicher finanzieller Aufwand.

Vorsicht ist auch bei sogenannten „Reinfleischdosen“ geboten. Viele Hundehalter übersehen hier, dass es sich lediglich um

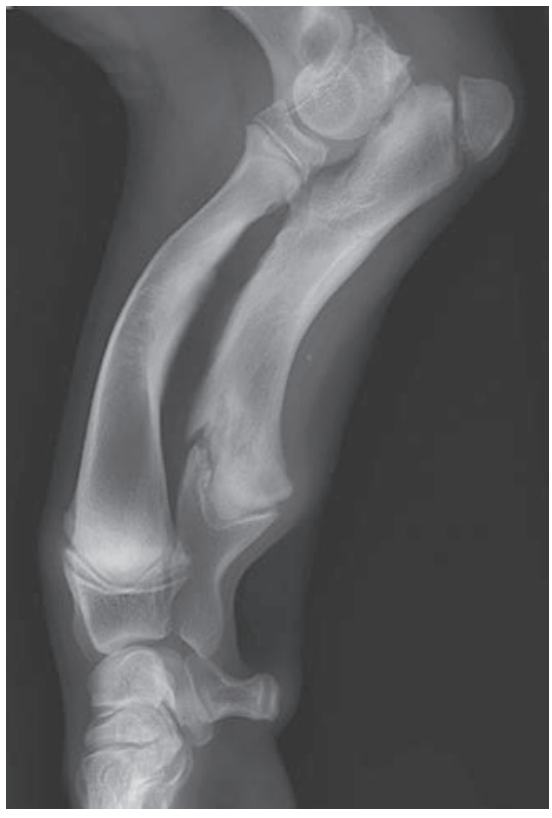

Abb. 2 Berner Sennenhund, männlich, 5 Monate alt. Radius-Curvus-Syndrom und damit einhergehende Außenrotation der Vordergliedmaßen infolge einer Kalziumüberversorgung. (c) PD Dr. Eberhard Ludewig, Klinik für Kleintiere, Universität Leipzig

ein Ergänzungsfuttermittel handelt, das in aller Regel nicht zusätzlich mineralisiert ist.

Fütterungsempfehlungen sind in der Regel auf der Verpackung nachzulesen. Hierbei ist unbedingt darauf zu achten, dass bei der Angabe der täglichen Futtermenge oftmals auf das zu erreichende Endgewicht und nicht auf das Momentangewicht Bezug genommen wird. Jedoch stellen auch diese nur Richtwerte dar. Die wirklich benötigte Futtermenge hängt von:

- Rasse

- Temperament

- täglichem Bewegungsangebot

des heranwachsenden Hundes ab. Bei Junghunden, die zu schnell an Körpermasse zulegen, ist die Energiezufuhr so zu reduzieren, dass deren Wachstum verlangsamt wird. Keinesfalls sollte je-

\section{konkret}

Kommt es zu einem untypischen Wachstumsverlauf (zu schnell, zu langsam), sind die deklarierten Futtermengen zu modifizieren. 
Tab. 4 Richtwerte zur Zusammensetzung von Trocken- und Feuchtalleinfuttern für wachsende Hunde (modifiziert nach [5]).

\begin{tabular}{|lll|}
\hline in $100 \mathrm{~g}$ & Trockenfutter $(1,6 \mathrm{M}) / 100 \mathrm{~g})$ & Feuchtfutter $(0,5 \mathrm{MJ} / 100 \mathrm{~g})$ \\
\hline $\mathrm{VRp}(\mathrm{g})$ & $17-18$ & $5-6$ \\
\hline $\mathrm{Rp}(\mathrm{g})$ & $20-23$ & $6-7$ \\
\hline $\mathrm{Ca}(\mathrm{g})$ & $0,8-1,2$ & $0,3-0,4$ \\
\hline $\mathrm{P}(\mathrm{g})$ & $0,6-1$ & $0,2-0,3$ \\
\hline $\mathrm{Na}(\mathrm{g})$ & $0,2-0,3$ & $0,06-0,1$ \\
\hline $\mathrm{K}(\mathrm{g})$ & $0,2-0,3$ & $0,06-0,09$ \\
\hline Zn (mg) & $9-10$ & 3 \\
\hline Se $(\mu \mathrm{g})$ & 11 & $3-4$ \\
\hline J $(\mu \mathrm{g})$ & $160-240$ & $30-70$ \\
\hline Vit. A (IE) & $800-1200$ & $160-300$ \\
\hline Vit. D (IE) & $70-90$ & $13-25$ \\
\hline Vit. E (mg) & $7-10$ & $1-3$ \\
\hline Vit. B1 (mg) & $0,2-0,3$ & $0,04-0,09$ \\
\hline
\end{tabular}

doch eine Futterrestriktion mit einem Verlust des Körpergewichts einhergehen.

Stellt sich anhand der Berechnung des Energiegehalts heraus, dass der heranwachsende Hund bisher energetisch überversorgt wurde, sollte er rund $10 \%$ weniger umsetzbare Energie im Vergleich zum Bedarf erhalten. In diesem Zusammenhang muss auch unbedingt die Fütterung sogenannter "Leckerchen“" hinterfragt werden. Gerade junge Hunde, die in einer intensiven Ausbildungs- und Trainingsphase sind, werden mit Snacks gefüttert und dabei wird ein erheblicher Teil ihres Energiebedarfs bereits abgedeckt. Gegebenenfalls muss also auch der Energiegehalt der Snacks berechnet und von der Gesamtration abgezogen werden. Dies gilt natürlich auch für die Erstellung eigener Rationen.

Abschließend wird in $>$ Tab. 4 ein Überblick über die empfohlene Zusammensetzung von Alleinfuttermitteln für wachsende Hunde zusammengestellt.

\section{Selbst zubereitete Rationen}

Die Zubereitung eigener Rationen stellt erhebliche Anforderungen an den Hundehalter. So ist auf ein ausgewogenes Protein:Energie-Verhältnis zu achten und eine bedarfsdeckende Vitamin- und Mineralstoffsubstitution vorzunehmen.

Tab. 5 Rationsbeispiel für eine heranwachsende Dogge (Adultgewicht: 60 kg KM).

\begin{tabular}{|c|c|c|c|c|}
\hline & \multicolumn{4}{|c|}{ Lebensmonat } \\
\hline & 3.-4. & 5.-6. & 7.-9. & 10.-12. \\
\hline Rindfleisch & $800 \mathrm{~g}$ & $850 \mathrm{~g}$ & $800 \mathrm{~g}$ & $900 \mathrm{~g}$ \\
\hline Vollei & $120 \mathrm{~g}$ & $120 \mathrm{~g}$ & $120 \mathrm{~g}$ & $120 \mathrm{~g}$ \\
\hline Reis & $600 \mathrm{~g}$ & $850 \mathrm{~g}$ & $900 \mathrm{~g}$ & 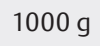 \\
\hline Möhre & $200 \mathrm{~g}$ & $250 \mathrm{~g}$ & $250 \mathrm{~g}$ & $250 \mathrm{~g}$ \\
\hline Öl & $10 \mathrm{~g}$ & $15 \mathrm{~g}$ & $15 \mathrm{~g}$ & $15 \mathrm{~g}$ \\
\hline Salz & - & - & $2,5 \mathrm{~g}$ & $3 \mathrm{~g}$ \\
\hline vitaminisiertes Mineralfutter & $30 \mathrm{~g}$ & $28 \mathrm{~g}$ & $20 \mathrm{~g}$ & $20 \mathrm{~g}$ \\
\hline Futterkalk & $18 \mathrm{~g}$ & $14 \mathrm{~g}$ & $10 \mathrm{~g}$ & $11 \mathrm{~g}$ \\
\hline
\end{tabular}


Eine präzise Anleitung zur Rationserstellung sollte in jedem Fall durch veterinärmedizinische Bildungseinrichtungen oder spezialisierte Tierärzte erfolgen.

Bei der dauerhaften Fütterung selbst zusammengestellter Rationen ist zu bedenken, dass Welpen in den ersten Monaten einer Nahrungsprägung unterliegen können, die sie für ihr ganzes Leben beibehalten. So ist es nicht selten zu beobachten, dass Welpen, die bisher nur mit selbstgekochten Rationen versorgt wurden, kommerzielle Alleinfuttermittel mit mangelnder Akzeptanz quittieren.

\section{Rationsgestaltung}

Wie soll nun solch eine Ration aussehen? Als Beispiel wird eine heranwachsende Deutsche Dogge mit einem Endgewicht von $60 \mathrm{~kg}$ vorgestellt und die notwendige Rationsanpassung im Laufe des 1. Lebensjahrs dargelegt $(\triangleright$ Tab.5).

Eine geeignete Ration sollte aus einer oder mehreren hochverdaulichen Rohproteinquellen bestehen. Hierbei kann es sich um Muskelfleisch handeln, aber auch Ei- oder Milchprodukte sind möglich. Die Fütterung von Leber oder anderen Innereien ist bei Einsatz eines vitaminisierten Mineralfuttermittels nicht nötig. Zudem enthält Leber z.T. sehr hohe Gehalte an Vitamin A und Kupfer, die von der Herkunft und Fütterung der Schlachttiere abhängig sind. Daher ist gerade beim wachsenden Hund eine Bedarfsdeckung (bzw. Über-/Unterversorgung) nicht kalkulierbar.

Pflanzliche Proteine, z.B. Sojaextraktionsschrot, können ergänzend zu tierischen Proteinquellen eingesetzt werden. Jedoch kann es durch einen erhöhten Gehalt an schwer verdaulichen Kohlenhydraten in pflanzlichen Futtermitteln zu Durchfällen und/oder Flatulenzen kommen. Daher sollte der Anteil an der Gesamtration $10 \%$ in der Trockensubstanz nicht übersteigen.

Als Energielieferanten eignen sich sowohl aufgeschlossene Kohlenhydrate (z.B. Getreideflocken, gekochter Reis und gekochte Kartoffeln/Nudeln) als auch tierische oder pflanzliche Fettquellen. Je nach Akzeptanz kann eine Gemüsequelle mitberücksichtigt werden, dies ist jedoch nicht zwingend notwendig.

Eine Ergänzung mit einem vitaminisierten Mineralfuttermittel ist grundsätzlich zu empfehlen, da eine bedarfsdeckende Versorgung nur über Einzelfuttermittel sehr aufwendig und aufgrund der schwerwiegenden Folgen einer Über- oder Unterversorgung gerade in diesem Alter nicht ratsam ist.

Vielfach unterliegen Besitzer der Fehlinformation, mit Milchprodukten den Kalziumbedarf des Hundes decken zu können. Dabei müssten extrem hohe Mengen Quark oder Joghurt gefüttert werden, was mit einer massiven Überversorgung mit Energie und Rohprotein einhergehen würde.

\section{BARF-Fütterung}

Zunehmend besteht auch unter Besitzern heranwachsender Hunde der Wunsch, ihre Tiere zu BARFen. Unter BARF (bones and raw foods) versteht man die Fütterung von rohem Fleisch (Muskelfleisch und Organgewebe) und fleischhaltigen Knochen mit der Ergänzung von rohem Obst/Gemüse, Ölen und ggf. verschiedensten Zusätzen.

$\mathrm{Zu}$ beachten ist hier in erster Linie, dass weder Schweinefleisch (kann Überträger der Aujetzky'schen Krankheit sein) noch Geflügelfleisch (häufige bakterielle Besiedlung mit Salmonellen und Campylobacter) roh angeboten werden sollten. Auch eine exakte Mineralstoffzufuhr, v.a. in Bezug auf ein ausgewogenes Ca:PVerhältnis, ist durch die Fütterung von Knochen nur schwer zu kontrollieren. Häufig werden Hunde mit BARF-Rationen massiv mit Protein überversorgt. Sie befinden sich im Gegenzug dazu vor allem mit kohlenhydratfreien Mahlzeiten energetisch eher im Bereich einer sehr knappen, z.T. deutlich bedarfsunterschreitenden Versorgung.

\section{Fütterungsregime}

Bei allen Fütterungsvarianten sollten Welpen mehrmals täglich gefüttert werden:

- im 3. Lebensmonat: 4-mal täglich
- im 4.-6. Lebensmonat: 3-mal täglich

- ab dem 7. Lebensmonat: 2-mal täglich

Die Futterzuteilung sollte unbedingt restriktiv erfolgen, um einen besseren Überblick über die aufgenommenen Futtermengen zu haben sowie eine Überversorgung mit Nährstoffen auszuschließen. Studien belegen, dass bei ad libitum gefütterten Welpen und Junghunden häufiger Gelenkdeformationen infolge von Wachstumsstörungen auftreten [2, 3, 5]. Besonders häufig fällt hierbei eine Außenrotation der Vordergliedmaßen auf.

In der Regel nehmen die Tiere bei ständig freiem Zugang zu Futter 125-135\% der eigentlich benötigten Futtermenge auf.

\section{Fazit}

Aufgrund der hohen Wachstumsrate und dem damit verbundenen hohen Risiko, Wachstumsstörungen zu entwickeln, stellt die Fütterung heranwachsender Hunde großer Rassen besondere Ansprüche an die Besitzer. Während die Fütterung von kommerziellen Alleinfuttermitteln für junge Hunde relativ problemlos möglich ist, setzen selbst zusammengestellte Rationen ein hohes Maß tierernährerischen Wissens beim Besitzer voraus. Hier ist es daher immer ratsam, die Ration von spezialisierten Tierärzten überprüfen zu lassen bzw. sich von ihnen einen geeigneten Rationsplan erstellen zu lassen.

Online zu finden unter

http://dx.doi.org/10.1055/s-0035-1547410

\section{Literatur}

Die Literatur ist in der Online-Version unter www.thieme-connect.de/products einsehbar.

\section{Cornelia Rückert}

PD. Dr. Ingrid Vervuert

Institut für Tierernährung, Ernährungsschäden und Diätetik

Veterinärmedizinische Fakultät

Universität Leipzig

An den Tierkliniken 9

04103 Leipzig

cornelia.rueckert@uni-leipzig.de 


\section{Literatur}

1 Blum JW, Zentek J, Meyer H. Untersuchungen zum Einfluss einer unterschiedlichen Energieversorgung auf die Wachstumsintensität und Skelettentwicklung bei wachsenden Doggen. J Vet Med (A) 1992; 39: 568-574

2 Dämmrich K. Relationship between nutrition and bone growth in large and giant dogs. J Nutr 1991; 121 (Supp. 11): 114-121

3 Dobenecker B, Kienzle E, Köstlin R et al. Mal- and overnutrition in puppies with and without clinical disorders of skeletal development. J Anim Physiol Anim Nutr 1998; 80: 76-81

4 Kamphues J. Supplemente zur Tierernährung. 12. Aufl. Hannover: Schaper; 2014

5 Meyer $\mathrm{H}$, Zentek J. Ernährung des Hundes. 7. Aufl. Stuttgart: Enke; 2013 